\title{
Norois
}

Environnement, aménagement, société

223 | 2012

Villes petites et moyennes

\section{Les villes moyennes françaises et leur rôle en matière d'aménagement du territoire : vers de nouvelles perspectives?}

The French medium-sized cities and their role in terms of spatial planning:

towards new perspectives?

\section{Frédéric Santamaria}

\section{OpenEdition \\ Journals}

Édition électronique

URL : http://journals.openedition.org/norois/4180

DOI : 10.4000/norois.4180

ISBN : 978-2-7535-2043-1

ISSN : 1760-8546

Éditeur

Presses universitaires de Rennes

\section{Édition imprimée}

Date de publication : 30 mars 2012

Pagination : 13-30

ISBN : 978-2-7535-2041-7

ISSN : 0029-182X

Référence électronique

Frédéric Santamaria, « Les villes moyennes françaises et leur rôle en matière d'aménagement du territoire : vers de nouvelles perspectives ? », Norois [En ligne], 223 | 2012, mis en ligne le 28 février 2014, consulté le 10 décembre 2020. URL : http://journals.openedition.org/norois/4180 ; DOI : https:// doi.org/10.4000/norois. 4180 


\title{
Les villes moyennes françaises et leur rôle en matière d'amé- nagement du territoire : vers de nouvelles perspectives?
}

\author{
The French Medium Sized Cities and their Role in Terms of Spatial Planning: \\ Towards New Perspectives?
}

\author{
Frédéric SANTAMARIA
}

UMR 8504 Géographie-cités, Laboratoire CRIA, (Université Paris 7 - Denis Diderot), Institut de géographie, 191 rue Saint-Jacques, 75005 Paris. (santamaria.f@free.fr)

\begin{abstract}
Résumé : Les villes moyennes ont participé aux évolutions économiques, démographiques, sociales et politiques des «Trente Glorieuses ». Au cours des années 1970, elles deviennent une catégorie de l'action publique. Depuis, on a assisté à un lent déclin de l'intérêt des pouvoirs publics pour cette catégorie de villes. Ceci peut s'expliquer par les évolutions économiques et spatiales de ces quarante dernières années. Depuis le début des années 2000, sous l'effet de la mondialisation, les responsables de la politique d'aménagement du territoire en France ont fait de la recherche de la compétitivité des territoires une orientation majeure. Dans ce contexte, et à cause de leur situation subalterne au sein de la division spatiale du travail et des difficultés qu'elles connaissent pour assurer le renouvèlement de leur base productive, il semble difficile de définir ce que pourrait être la place de cette catégorie de villes en matière d'aménagement du territoire. Or, aujourd'hui, ces villes sont concernées par certains enjeux d'aménagement du territoire comme la promotion d'une organisation polycentrique, la structuration des espaces métropolisés ou la question des rapports urbain/ rural. Ainsi, au-delà des changements économiques et spatiaux, ce sont les caractéristiques communément admises de la catégorie des villes moyennes qui constituent aujourd'hui un obstacle à leur reconnaissance comme outils d'aménagement du territoire.
\end{abstract}

Abstract: In France, medium-sized cities participated to the period of fast growth of the post Second World War ("Trente Glorieuses") in economic, demographic, social and political terms. During the 70s, they become a category of public action. Since then, there has been a slow decline in the interest of the government for this category of cities. This can be explained by changes in economic and space of the last forty years. Since the early 2000s, as a result of globalization, regional competitiveness has become a major focus for policy makers in the spatial planning field. In this context, and because of their subordinate position within the spatial division of labor and the difficulties they face in ensuring the renewal of their productive base, it seems difficult to define what might be the role of this category of towns in terms of spatial planning. Yet today, these cities are affected by specific issues of spatial planning: the promotion of a polycentric organization, the structuration of metropolised spaces or the issue of urban / rural relations. Thus, beyond the economic and spatial changes, it is the commonly accepted characteristics of the category of towns that now constitute an obstacle to their recognition as tools for spatial planning.

Mots clé : villes moyennes - aménagement du territoire - France

Keywords: medium-sized cities - spatial planning - France 


\section{INTRODUCTION}

Les villes moyennes constituent un objet d'étude incommode dont la définition doit être remise sur le métier en fonction du contexte géographique au sein duquel elles sont étudiées, d'évolutions socio-économiques susceptibles de caractériser cette catégorie de villes et, également, de l'attention que portent les pouvoirs publics à cet ensemble de villes. Dans ce sens, les éléments de leur définition peuvent varier d'une étude à l'autre. Cependant, comme toutes les villes, les villes moyennes sont définies selon des critères de taille, des critères fonctionnels, des caractéristiques socio-économiques et des représentations. Dans le cas français, à l'échelle nationale, on s'accorde généralement sur des critères de taille, sur leur rôle fonctionnel et sur les grands traits de leur évolution socio-économique de la période des «Trente Glorieuses » à nos jours. Ainsi, les villes moyennes sont généralement situées dans une fourchette de taille comprise entre 20000 et 200000 habitants ${ }^{1}$. Leur rôle est celui de la desserte d'un espace infrarégional dépassant cependant le rayonnement strictement local. Leurs évolutions socio-économiques sont référées à celles de l'appareil de production dans la période d'après-guerre. Elles sont présentées comme des lieux privilégiés de la production de type fordiste durant la période de forte croissance économique de l'après-guerre puis comme des lieux particulièrement touchés par les crises industrielles successives à partir des années 1970; situation qui pose, encore aujourd'hui, la question de leur place économique au sein du territoire national et par rapport à la concurrence internationale des territoires étrangers. Ces éléments de définition s'accompagnent d'une représentation des villes moyennes françaises particulière, celle que l'on peut résumer par l'expression de villes « où il fait bon vivre...». Enfin, à partir des années 1970, dans le domaine de l'aménagement du territoire, les villes moyennes

1. On fait référence aux unités urbaines. Il s'agit ici des limites extrêmes de la catégorie qui peuvent varier selon les auteurs et les organismes en charge de les étudier. Cependant, on pourra trouver des limites plus resserrées ou plus larges de cette catégorie de villes. Le recours à l'aire urbaine a conduit la DIACT, dans son étude de 2007 sur les villes moyennes, à identifier des aires urbaines de villes moyennes allant de 30000 à 200000 habitants : « La DIACT a choisi de retenir l'aire urbaine pour appréhender les villes moyennes en sachant qu'une commune de 20000 habitants dispose d'une aire urbaine de l'ordre de 30000 habitants et une commune de 100000 habitants d'une aire urbaine avoisinant les 150000 habitants. Ce seuil a été élevé à 200000 habitants, seuil qui constitue généralement le plancher pour la strate des grandes villes » (DIACT, 2007, p. 5) font l'objet de l'attention des pouvoirs publics. Elles deviennent alors une catégorie de l'action publique. Cependant, depuis cette époque, on a assisté à un lent déclin des actions d'aménagement du territoire en faveur de cette catégorie de villes. Ceci peut s'expliquer par les évolutions économiques et spatiales de ces quarante dernières années. En effet, depuis le début des années 2000, sous l'effet de la mondialisation, les responsables de la politique d'aménagement du territoire en France ont fait de la recherche de la compétitivité des territoires une orientation majeure. Dans ce contexte, et à cause de leur situation de dépendance au sein de la division spatiale du travail et des difficultés qu'elles connaissent pour assurer le renouvèlement de leur base productive, il semble difficile de définir ce que pourrait être la place de cette catégorie de villes en matière d'aménagement du territoire. Pour autant, aujourd'hui, ces villes sont concernées par certains enjeux majeurs d'aménagement du territoire comme la promotion d'une organisation polycentrique des territoires, la structuration des espaces métropolisés ou la question des rapports urbain/rural. Partant, nous entendons démontrer, qu'au-delà des changements économiques et spatiaux, ce sont les caractéristiques communément admises de la catégorie des villes moyennes qui constituent un obstacle à leur reconnaissance comme outils d'aménagement du territoire.

\section{DES VILLES Disqualifiées POUR L'AMÉNAGEMENT DU TERRITOIRE}

Alors que les villes moyennes sont apparues comme une catégorie légitime de l'action publique dans les années 1970 du fait de leur participation multiforme aux évolutions socio-économiques et spatiales de la France, les difficultés qu'elles rencontrent à partir de la fin des années 1970 en font une catégorie disqualifiée de l'aménagement du territoire.

\section{Des villes ayant participé aux mutations de la société française de l'après-guerre aux années 1970}

Les villes moyennes ont pris une part très active aux mutations tant économiques, spatiales, sociales que politiques qui concernèrent l'ensemble de la 
société française dans la période d'après guerre jusqu'aux années 1970. En effet, dans cette période, elles ont participé au mouvement général d’industrialisation et d'urbanisation. Elles ont également été des lieux de modernisation économique, sociale et politique.

\section{Participation à la modernisation économique}

Dans leurs travaux portant sur l'évolution des structures socio-économiques du système urbain français de 1954 à 1975, D. Pumain et Th. SaintJulien (1978) montrent qu'au début de la période, les «villes de tailles intermédiaires (les auteurs considèrent ici les villes de 60000 à 300000 habitants) représentaient le point faible de l'armature urbaine française en matière d'industrialisation » (p. 128). Mais, entre 1954 et 1962, l'industrialisation touche particulièrement ces mêmes agglomérations; ensuite, entre 1962 et 1968, ce sont les villes de 40000 à 50000 et de 20000 à 30000 habitants qui sont à leur tour concernées. L'épanouissement du modèle fordiste dans cette période oblige les entreprises (spécialement les entreprises industrielles) à trouver de nouvelles sources de main d'œuvre. Or, les coûts liés à l'extension dans les zones d'implantation initiale, les différentiels de rémunération et les problèmes généraux que font naître la concentration des activités (congestion, risques sociaux), favorisent l'implantation d'unités industrielles nouvelles dans les villes moyennes dont l'environnement, le plus souvent rural, permet de disposer de réservoirs de main d'œuvre importants issus de l'exode rural ${ }^{2}$. Cette période de décentralisation industrielle se poursuivra jusque dans les années 1970. Les villes moyennes voient alors s’installer des unités de production de grands groupes nationaux et internationaux ${ }^{3}$.

\section{Participation à l'urbanisation}

Sur la période 1954-1975, les villes moyennes connaissent une croissance démographique soutenue qui s'inscrit dans un processus plus général d'urbanisation du pays (tableau 1).

De 1954 à 1962, les trois classes considérées, de 20000 à 50000 habitants, puis de 50000 à 100000 habitants, et enfin de 100000 à 200000 habitants, connaissent des taux de variations annuels moyens de leur population supérieurs à l'augmentation de la population urbaine totale sur la même période $(+1,86 \%)$. Aucune autre catégorie de villes ne fait mieux que celles qui sont alors comprises entre 50000 et 200000 habitants. Seules les villes de plus de 200000 habitants dépassent de peu la classe de villes moyennes allant de 20000 à 50000 habitants. De la même manière, entre 1962 et 1968, les différentes classes de villes moyennes voient leur population progresser plus fortement que la population urbaine totale du pays $(+1,79 \%)$. À quelques dixièmes prés, en plus ou en moins,

\begin{tabular}{|c|c|c|c|}
\hline $\begin{array}{c}\text { Classes des tailles } \\
\text { des agglomérations }\end{array}$ & $1954-1962$ & $1962-1968$ & $1968-1975$ \\
\hline Moins de 5000 & 0,88 & 1,16 & 1,15 \\
\hline $5000-10000$ & 1,21 & 1,43 & 1,11 \\
\hline $10000-20000$ & 1,6 & 1,76 & 1,28 \\
\hline $20000-50000$ & 1,88 & 2,03 & 1,52 \\
\hline $50000-100000$ & 2,16 & 2,06 & 1,63 \\
\hline $100000-200000$ & 2,54 & 2,31 & 1,17 \\
\hline Plus de 200000 & 2 & 2,13 & 0,53 \\
\hline Agglomération parisienne & 1,88 & 1,3 & 1,14 \\
\hline Ensemble des unités urbaines & 1,86 & 1,79 & \\
\hline
\end{tabular}

Tableau 1 : Taux de variation moyens annuels de la population des agglomérations par classe de taille (en \%) de 1954 à 1975 (source : Santamaria, 1999)

Average annual rate of change of urban areas population by size class (in \%) from 1954 to 1975
2. Rappelons qu'en 1946, plus d'un tiers de la population active était employée dans l'agriculture.

3. Comme Goodyear, Valeo, Jaeger et Procter and Gamble à Amiens, Citroën dans l'agglomération de Caen, Renault à Limoges, Deutz et Mannesmam à Châlons-sur-Marne (Châlons-en-Champagne). 
elles égalent la progression des plus grandes villes. De 1968 à 1975, alors que la population urbaine totale augmente moins vite $(+1,14 \%)$, leurs taux de variation annuels moyens sont toujours supérieurs à la progression générale des unités urbaines. Seule la classe inférieure de la catégorie des villes moyennes, de 20000 à 50000 habitants, connaît un taux un peu inférieur à celui de la classe des villes comprises entre 10000 et 20000 habitants. Les plus grandes villes moyennes comprises entre 100000 et 200000 habitants étant celles qui, sur les trois périodes considérées, enregistrent les taux de croissance les plus forts. Enfin, il faut noter que les taux de variation annuels moyens de l'agglomération parisienne ne dépassent jamais ceux des classes de villes moyennes présentées ci-dessus. Par conséquent, sur ces trois périodes intercensitaires, les villes moyennes participent fortement au mouvement général de progression de la population urbaine lié à plusieurs phénomènes alors concomitants : un accroissement naturel assez soutenu, l'apport démographique que constituent l'immigration et l'exode rural. Sur ce dernier point, le mouvement de délocalisation industrielle vers les villes moyennes les place dans une position de réceptacle majeur des populations issues des campagnes ${ }^{4}$. Dans son ouvrage de 1974, le Doyen Lajugie remarque que les villes moyennes ont enregistré, pendant la période d'après-guerre, des excédents migratoires importants, relativement plus élevés (en pourcentage) et à peine inférieurs en valeur absolue à ceux des grandes villes françaises et de l'agglomération parisienne. Ces excédents constituent par ailleurs la première source d'accroissement de la population devant celui généré par les excédents naturels. Entre 1954 et 1962, alors que les villes moyennes connaissent des soldes migratoires négatifs avec les grandes agglomérations et Paris (-100000 habitants), ceux-ci sont positifs avec les petites villes $(+86000$ habitants $)$ mais surtout avec les communes rurales (+304000 habitants) (Lajugie, 1974). Les villes moyennes ont donc contribué sur cette période à fixer une partie des populations qui se seraient dirigées vers Paris ou vers les grandes villes (Pitié, 1971).

4. Il ne faut cependant pas négliger l'impact du phénomène général de tertiarisation de l'économie française également susceptible, du fait des créations d'emplois qu'il implique, d'attirer vers ces villes des populations nouvelles.

\section{Participation à la modernisation sociale et politique}

Pour N. Commerçon (1990), « l'essentiel du changement apparu $[\ldots]$ se mesure par les mutations sociales survenues » en villes moyennes qui s'affirment alors comme « de véritables plaques tournantes de la société tout entière » (p. 3). Deux phénomènes majeurs conduisent à cette situation : l'attraction des populations rurales, qui se traduit par un rajeunissement des structures démographiques, et la modification des structures économiques, le plus souvent dans le sens d'un renforcement des activités industrielles et de services à l'usage des populations. Ces deux mouvements conduisent à l'augmentation de la part des cadres surtout moyens, des ouvriers et des employés souvent non qualifiés. Les villes moyennes offrent alors aux populations issues de l'exode rural l'accès au niveau inférieur des classes moyennes, en leur proposant des emplois stables à des niveaux de qualification faible et intermédiaire. Cette situation fait écho à la progression de la catégorie des classes moyennes dans la période d'après-guerre ${ }^{5}$. Ces modifications socio-économiques correspondent également à la diffusion d'un modèle de société. Elles se traduisent par un gonflement démographique qui implique la création d'équipements, notamment de type commercial, pour satisfaire les populations nouvellement installées. De ce fait, les villes moyennes et ses habitants se convertissent aux spécificités de la vie urbaine: déplacement en voiture individuelle, disjonction entre le lieu de travail et celui de résidence, achats groupés dans les grandes surfaces, accès aux loisirs organisés, etc. Ainsi, à l'instar de ce qui se passe dans les grandes villes, l'on assiste à une spécialisation des espaces des villes moyennes (habitation, production, consommation, loisir...) et, pour les populations installées dans ces villes, à un véritable changement de modes de vie.

Selon Mabileau et Sorbets (1989), le changement social dans les villes moyennes se traduit également par un changement politique. Ce dernier s'exprime avec force lors des élections municipales de 1977 qui se traduisent par le renouvellement des équipes, un investissement dans une structuration bureau-

\footnotetext{
5. Suivant la répartition des classes sociales, adaptée aux réalités sociales de l'après-guerre, proposée par P. Courtheoux et F. Martos cités dans F. Braudel et E. Labrousse (1993) les classes moyennes représentent 40,6\% de la population en 1975 contre $29,7 \%$ en 1954 .
} 
cratique des administrations locales et de nouveaux modes de gestion marquée à la fois par la politisation («vague de gauche») et le développement d'une technocratie locale.

\section{Des villes en difficulté}

Les mutations économiques qui surviennent au cours des années 1970 ébranlent les bases industrielles sur lesquelles était fondé le développement des villes moyennes dans la période des "Trente Glorieuses » et remettent en cause leur place dans le processus de production national. Au-delà, les villes moyennes françaises doivent affronter une concurrence croissante entre villes pour laquelle elles semblent peu préparées.

\section{Des bases économiques fragilisées}

On aurait pu penser que le développement industriel des villes moyennes impliquerait une spécialisation relative de ces villes dans un type donné de tâches. Ce fut le cas, mais l'on a surtout assisté à leur cantonnement dans une position et dans des fonctions subalternes au sein du processus de production. Cela signifie que la spécificité des implantations industrielles en villes moyennes s'est surtout résumée à la recherche de main d'œuvre disponible du fait de l'exode rural, docile et peu formée, offrant des différentiels de salaire de 30 à $50 \%$ avec la région parisienne (Sallez, 1992); une main d'œuvre à laquelle furent essentiellement confiées des tâches d'exécution. Finalement, on assiste à une « division spatiale du travail » distinguant les lieux de conception de ceux de production. Dans ces conditions, les sièges sociaux et les fonctions les plus élevées se trouvent dans les grands centres. Les villes secondaires, ainsi que les plus petites, assurent les tâches de mise en ouvre et d'exécution de la production. Pour comprendre ce processus, il faut le replacer dans le contexte de l'économie française de la période considérée. Les villes moyennes constituent alors un des éléments du modèle fordiste de développement. Outre la division des tâches, les ateliers et les usines situés en villes moyennes sont soumis à une organisation verticale qui les prive de tout pouvoir d'initiative, de toute capacité de commandement. Selon Michel (1987), c'est ce modèle de développement qui marque leur « commune appar- tenance à l'espace d'exécution ». Dés lors, se posait la question de savoir quelle base de développement à venir pouvait pérenniser une telle industrialisation. La crise économique de 1974 qui a marqué le début d'une profonde remise en question du modèle fordiste se traduit par l'effondrement de pans entiers de la production traditionnellement situés en villes moyennes, comme ceux de la mécanique légère, de l'appareillage électrique ou de la confection. Rétrospectivement, on peut affirmer avec Loinger (1994), que les « délocalisations industrielles effectuées dans les années 1960-1970 sur un mode taylorien de répartition des tâches à l'échelle nationale ont souvent eu des effets pervers au sens où les unités de production, concentrées sur la pure et simple exécution des tâches, n'ont guère contribué à renforcer le potentiel industriel local du point de vue de l'autonomie du système économique local ». Encore aujourd'hui, certaines villes moyennes sont durement affectées par des fermetures d'entreprises et certaines voient leur emploi industriel dans des secteurs tels que ceux de l'équipement automobile, de la mécanique, du caoutchouc, du textile et de la chaussure continuer à décliner.

Cependant, les villes moyennes ne sont pas les seules à subir les effets des mutations économiques de cette période. Certains grandes villes industrielles du Nord de la France (Valencienne, Béthune, Lens) ont été durement touchées par des crises sectorielles alors que certaines villes moyennes, disposant d'un secteur tertiaire porteur et d'industries dynamiques (Annecy), ont connu des évolutions beaucoup plus favorables. Par conséquent, au-delà des effets des mutations économiques qui affectent durement certaines villes moyennes jusqu'à aujourd'hui, c'est la place de cette catégorie de villes dans le processus de production qui pose problème.

\section{La question du modèle de développement économique des villes moyennes}

L'intégration des villes moyennes dans un processus de production hiérarchique se trouve remise en cause par les nouvelles exigences de la production : celles de la réactivité, peu favorisée par des organisations hiérarchiques; de la compétitivité opposée à la simple productivité fordiste; d'une organisation de la production à flux tendus opposée aux prévisions d'accroissement constant de la production. Ces villes 
ne semblent plus fournir un attrait spécifique pour la production industrielle, notamment en termes de recherche d'une main d'œuvre qualifiée également mobilisée dans les secteurs tertiaires dynamiques. En outre, leur cantonnement dans des tâches d'exécution a restreint leur aptitude à l'innovation et à la création d'activités et de produits, arguments susceptibles de permettre aux villes de retenir les activités existantes, voire d'en attirer de nouvelles. Ainsi, ce qui a pu apparaître comme l'âge d'or du développement industriel des villes moyennes (Dodier, 1995), des années 1960 jusqu'au milieu des années 1970, est aujourd'hui considéré comme un élément pesant sur l'avenir de ces villes. Dans l'ensemble, des mesures relatives à l'évolution de l'emploi effectuées à l'échelle des aires urbaine de 30000 à 200000 habitants montrent que les catégories considérées ne se distinguent guère des évolutions enregistrées par l'ensemble des aires urbaines françaises entre 1990 et 1999. En effet, sur cette période, la variation d'emploi de ces aires urbaines se situe dans la moyenne nationale ${ }^{6}$. En outre, les aires urbaines en question ont connu, au cours des années 1990, une tertiarisation de l'emploi qui peut présager d'une réorientation économique de cette catégorie de villes (DIACT, 2007).

Cependant, le sens de ces évolutions reste incertains car cette tertiarisation de l'emploi ne semble pas permettre d'envisager une insertion de ces villes dans les grands flux de développement économique du fait de la nature des activités tertiaires concernées (ex : administration, commerce de détail, services à la personne, éducation, santé, aide sociale $)^{7}$. Partant, l'interrogation sur l'avenir économique des villes moyennes reste d'actualité depuis maintenant une vingtaine d'années. En 1995, dans une communication au colloque de Cholet sur les villes moyennes, Dodier (1995) s'interrogeait sur la nature de la transition économique des villes moyennes de l'après fordisme. En 2007, dans un ouvrage consacré aux villes moyennes par la DIACT, les villes moyennes étaient présentées comme une catégorie de villes se situant à la croisée de la tradition

6. Sur la période 1990-1999, la variation de l'emploi total au niveau de l'aire urbaine se situe un peu au-delà de la moyenne nationale des aires urbaines $(+3,8)$ pour les aires urbaines situées dans la catégorie de 30000 à 200000 habitants. Cela concerne les strates comprises entre 30000 et 50000 habitants $(+4,4 \%), 50000$ et 100000 habitants $(+4,2 \%)$ et 100000 à 200000 habitants (+ $4 \%)$ (DIACT, 2007).

7. On consultera à ce sujet le tableau $n^{\circ} 3$ « Les secteurs les plus créateurs et destructeurs d'emplois dans les villes moyennes entre 1993 et 2001 » dans DIACT, 2007. industrielle et de la transformation de l'économie des services (DIACT, 2007). Ces perspectives économiques incertaines posent également la question de la place des villes moyennes vis-à-vis des autres villes et particulièrement vis-à-vis des villes situées au niveau supérieur de la hiérarchie urbaine.

\section{Une position délicate au sein du réseau urbain français}

Le mouvement de mondialisation a fait prendre conscience de la nécessité pour les villes françaises de penser leur avenir autrement qu'en termes nationaux. Ce changement d'échelle fait qu'il est difficile d'envisager que les villes moyennes se placent en bonne position dans la compétition internationale. Elles sont comme exclues, a priori, du nouveau système concurrentiel. En 1989, l'enquête menée pour la DATAR par R. Brunet (1989) sur la base de seize indicateurs concernant le poids démographique des villes, leur puissance économique, la qualité de leurs infrastructures de communication, l'intensité de leurs activités de recherche et de haute technologie, leurs fonctions culturelles et leur notoriété, a permis de dégager ce que l'auteur appelait les villes de «taille européenne ». Le travail portait sur les villes de plus de 200000 habitants, mais l'auteur reconnaissait que les villes de l'ordre de 200000 ou 250000 habitants n'avaient pas réellement « la taille européenne ». De manière générale, les éléments tels que le degré de diversification des activités économiques, le niveau des équipements, le degré de qualification de la main d'œuvre, l'insertion dans des réseaux d'échanges nationaux et internationaux dépendent de la taille des villes. Or, seules les plus grandes d'entre-elles sont capables de mobiliser suffisamment de ressources dans ces domaines pour participer aux échanges internationaux. Finalement, ce sont moins les difficultés des villes moyennes qui importent, quand on considère leur avenir au sein de réseau urbain, que la pertinence même de cette taille de ville dans le contexte de concurrence internationale.

\section{Du point de vue de l'action publique : d'un intérêt ambigu à un relatif désintérêt}

Dans le cadre de la politique d'aménagement du territoire, l'État s'engage, à partir de 1972, dans une 
politique d'aide aux villes moyennes considérant qu'elles accueillent le plus gros de la croissance urbaine. Ces villes apparaissent alors comme un modèle alternatif aux problèmes engendrés par la croissance urbaine des grandes agglomérations. On insiste en particulier sur le fait qu'elles disposent d'un cadre de vie plus accueillant au sein duquel peut se concevoir une vie urbaine plus agréable. Enfin, l'apport de nouvelles populations fait prendre conscience aux pouvoirs publics que ces villes doivent répondre correctement aux besoins de leurs habitants en leur offrant des logements, ainsi que des conditions de vie et de formation favorables à un bon accueil des migrants issus du monde rural (Lajugie, 1974). Une véritable politique des villes moyennes est inaugurée par la DATAR en 1973. Elle met notamment l'accent sur des opérations d'embellissement et d'aménagement du cadre de vie. On peut donc dire que les villes moyennes se voient intégrées, en tant que catégorie urbaine, à la politique nationale d'aménagement du territoire. Toutefois, la politique des villes moyennes a ceci d'ambigu qu'elle correspond plus volontiers à des actions en faveur du développement urbain qu'à une véritable politique globale d'aménagement du territoire national. En effet, alors que la politique des métropoles d'équilibre avait pour ambition de structurer l'espace régional en offrant à la France des pôles d'importance, alternatifs à l'expansion parisienne, la politique des villes moyennes se déploie à travers des projets d'aménagement d'équipements publics ou le développement des services collectifs centrés sur les besoins des villes elles-mêmes (Dumas, 1991). Tout se passe comme si les villes moyennes ne pouvaient être considérées comme des éléments actifs de l'aménagement du territoire mais plutôt comme des lieux de régulation de tendances concernant la société dans son ensemble ${ }^{8}$.

Au cours des années 1980, le souci de l'État est moins d'établir des politiques nationales d'aménagement du territoire que de gérer les conséquences les plus douloureuses de la crise économique dans un contexte difficile pour les finances publiques. Pour les villes moyennes, cela se traduit, dès 1976, par une réorientation des financements des opérations concernant l'environnement urbain vers l'attraction de nouvelles activités industrielles et ter-

8. Pour une approche critique de la politique des villes moyennes, on consultera M. Michel (1977). tiaires, grâce à des primes à l'installation. En 1979, la politique des villes moyennes est définitivement abandonnée. Pour parer aux désengagement financier de l'État, les collectivités locales, au premier rang desquelles les villes, tentent par elles-mêmes d'assurer leur prospérité. On assiste alors à une véritable compétition entre elles dans le but d'attirer de nouveaux investissements publics et privés. En termes d'aménagement du territoire, la concurrence entre villes est non seulement admise par l'État, mais elle fait l'objet d'un réel encouragement. En 1986, le rapport Guichard sur l'aménagement du territoire consacre la notion de concurrence entre villes qui s'exerce dorénavant au moins à l'échelle européenne (1986). L'auteur du rapport considère que le sort des villes se mesure dorénavant en termes d'attractivité et de créativité relative. C'est donc la capacité d'innovation des villes qui est dorénavant en jeu à travers, notamment, la présence d'une main d'œuvre formée pour utiliser les technologies innovantes. Pour ce faire, outre le développement de capacités localisées de formation et de recherche, les villes doivent répondre aux exigences de cette main d'œuvre en termes de services et de cadre de vie. Ces démarches destinées aux populations locales doivent aussi assurer la promotion de l'image de la ville à l'extérieur de manière à la rendre plus attractive. Elles sont donc soutenues par des politiques de communication visant à donner une image avantageuse et attrayante de la cité mettant en avant ses atouts spécifiques. Enfin, il ne servirait à rien de disposer de ces atouts si les personnes et les entreprises ne pouvaient se déplacer ou assurer la mobilité de leurs employés dans le cadre d'une économie mondialisée. L'équipement en termes de dessertes par les grands réseaux de communication matériels et immatériels constitue également un aspect tout à fait discriminant du développement urbain. L'ensemble de ces éléments est dorénavant un passage obligé pour les villes qui entendent participer à la concurrence urbaine nationale et internationale. Cette approche relève d'une conception concurrentielle et élitiste de l'aménagement du territoire qui profite aux villes répondant aux nouvelles exigences; c'est-à-dire, essentiellement, les plus grandes d'entre-elles. Dans ce contexte, la question du rôle des villes moyennes en matière d'aménagement du territoire se pose à nouveau. 
La politique des réseaux de villes inaugurée par la DATAR en 1990 apparaît alors comme une volonté de promouvoir les villes moyennes en matière d'aménagement du territoire dans ce nouveau contexte. En effet, cette politique s'adresse alors principalement aux villes moyennes ${ }^{9}$. L'État entend favoriser la libre association de villes désireuses de mettre en commun des moyens et de réaliser des actions ensemble. La politique des réseaux de villes devait permettre aux villes y participant de franchir des effets de seuil, de réaliser des économies d'échelles en groupant leurs forces, d'assurer des complémentarités (en termes d'équipements, de services...), et d'atteindre ainsi une taille critique pour affronter dans de meilleures conditions la concurrence urbaine. Cette politique qui visait à assurer une meilleure intégration des villes françaises, et, partant, du territoire national à l'Europe, est alors présentée comme un élément susceptible de répondre aux préoccupations en termes d'aménagement du territoire (DATAR, 1995). Cependant, les résultats des réseaux de villes étant jugés décevants en termes de réalisations concrètes (Tesson, 1996), cette politique est peu à peu délaissée par l'État ${ }^{10}$ alors même que, depuis 2004, cette logique de mise en réseau des villes a été mobilisée en faveur de la strate supérieure du réseau urbain à travers la politique nationale de coopération métropolitaine ${ }^{11}$. Cette politique vise à consolider le rayonnement des métropoles françaises au niveau européen en soutenant l'émergence et le renforcement de leurs coopérations. Aujourd'hui, elles concernent 15 ensembles métropolitains, dont certains incluent des villes de taille moyenne ${ }^{12}$ (Arras, Cambrai, Maubeuge, Hénin, Caen ${ }^{13}$, Thionville, Épinal, Belfort, Montbéliard, Besançon ${ }^{14}$, Annecy, Chambéry, Valence, Roanne, Bourg-en Bresse,

9. F. Tesson (1996) dans sa thèse consacrée aux réseaux de villes en 1996 dénombrait 18 réseaux de villes dont 8 associant uniquement des villes moyennes, 3 associant des villes moyennes et des petites villes et 7 associant des villes moyennes et des grandes villes. Sur 63 villes concernées, 47 étaient des villes moyennes (on dénombrait 11 grandes villes et 5 petites villes).

10. Les réseaux de villes ont pu être financés jusqu'en 2006 au titre du volet territorial des contrats de plan État-Région (circulaire du 5 juin 2000 relative à la politique des réseaux de villes).

11. Les coopérations métropolitaines peuvent s'organiser soit sur une stricte logique de réseau, soit à partir d'espaces contigus, soit dans une forme d'entre deux.

12. Les villes énumérées correspondent à des villes entre 20000 et 200000 habitants au sein de l'unité urbaine.

13. Il s'agit d'une capitale régionale. Elle est considérée ici comme une ville moyenne par la taille (cf. supra note ci-dessus).

14. Cf. supra note ci-dessus.
Vichy, Le Puy-en-Velay, Aurillac, Montauban, Albi, Castres-Mazamet, Auch, Saint-Nazaire), organisés autour de grandes villes le plus souvent métropoles régionales (Lille, Rennes-Nantes-Angers, Rouen-Le Havre, Toulouse, Clermont-Ferrand, Lyon-Saint-Étienne, Marseille, Nice, Toulon, Dijon, Strasbourg, Metz-Nancy, Grenoble) ou de quelques villes moyennes assurant, du côté français, la structuration d'espaces frontaliers (DunkerqueCalais-Boulogne sur Mer-Saint-Omer; ForbachSarreguemines). Certaines villes moyennes peuvent donc faire l'objet, bien qu'indirectement, d'une politique nationale pour autant qu'elles soient situées dans l'orbite d'une métropole régionale éligible au titre de la coopération métropolitaine ou dans des espaces stratégiques du point de vue des relations transfrontalières européennes.

Ce tableau de l'évolution des politiques d'aménagement en rapport avec les villes moyennes doit être complété par la présentation de l'action de l'État en matière d'implantations universitaires au cours de la première moitié des années $1990^{15}$. Ces années sont marquées par la volonté de développer sur le territoire national une offre universitaire nouvelle visant explicitement à un rééquilibrage qualitatif et quantitatif entre l'île-de-France et les régions et entre les grandes villes et les villes moyennes. L'objectif du plan Université $2000^{16}$ (1990-1995) était à la fois de faire face à la forte croissance des effectifs universitaires et de développer, dans une logique de proximité, une offre universitaire en rapport avec les territoires d'insertion. À l'issue de la période, outre les sites en villes moyennes préexistants, le territoire national se couvre de structures universitaires dont les lieux d'implantation privilégiés sont les villes moyennes comme le montre la carte cidessous (figure 1) qui présente les sites proposant des formations dans les domaines scientifiques et techniques plus particulièrement visés par le plan Université 2000.

Cependant, dès la fin des années 1990, marquées par la stabilisation des effectifs universitaires et la prévision de leur décroissance, la question de

15. On pourrait également évoquer la politique des systèmes productifs locaux (SPL) lancée en 1998 par la DATAR mais cette politique, centrée sur les entreprises, n'était pas destinée directement aux villes moyennes. Cependant, elles ont pu être concernées par cette politique dans la mesure où elles se trouvaient situées au sein d'un territoire des SPL labellisés.

16. Le plan «Université du $3^{e}$ millénaire », lancé en 1999, concernait surtout des réhabilitations. 


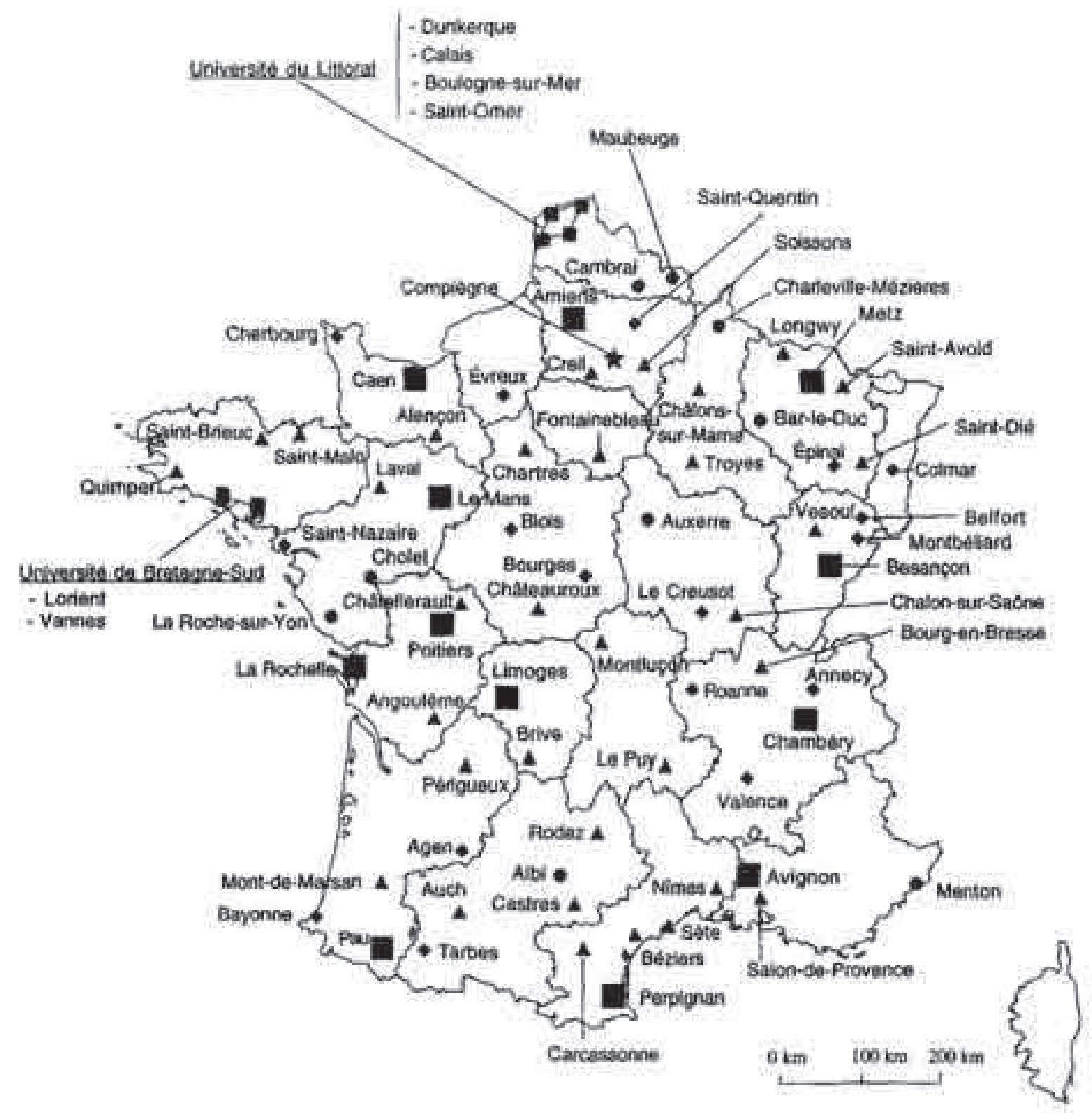

Valles sięges d̛univenites

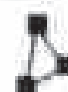

Villesporricipant a des universites multipolaites

* Villes sieges if faniversith de technologie

- Viffex disposans dfintennes universitaires et drut

- Villes disposan moiquement drantennes nuivecuitaires

a Villes disponat animaenent oriuT

Figure 1: Villes moyennes disposant de structures universitaires dans les domaines scientifiques à l'issue du plan « Université 2000 » (source : Santamaria, 1999)

Medium-sized cities that have university structures in science at the end of the plan "University 2000" 
l'avenir de ces implantations universitaires se pose. La réforme des universités lancée en 2007 a également suscité nombre d'interrogations de la part des acteurs des villes moyennes notamment par rapport aux objectifs affichés de constitution de pôles de renom international qui pourraient se traduire par une réorientation des étudiants et des moyens vers ces pôles. Les expérimentations conduites dans le domaine universitaire par la DATAR dans 8 villes moyennes lauréates d'un appel d'offres lancé en 2007 montrent que les acteurs de ces villes sont particulièrement préoccupés par la place de l'offre universitaire locale vis-à-vis des effets de polarisation vers les métropoles régionales ${ }^{17}$. Toujours au titre de cette expérimentation, on constate que si les acteurs locaux des villes moyennes dans le domaine de l'enseignement supérieur et de la recherche ont été amenés à réfléchir sur leur place dans le contexte universitaire régional et national, l'intérêt de l'État, des collectivités régionales et des grands acteurs universitaires ne s'est pas encore manifesté avec force ${ }^{18} \ldots$

À l'issue de ces développements sur la place des villes moyennes vis-à-vis de l'action en matière d'aménagement du territoire, on peut constater un relatif désintérêt pour cette catégorie de villes. Ceci peut s'expliquer, comme nous l'avons vu, par les évolutions même de cette catégorie au sein du réseau urbain français et également par un repositionnement de l'action, inauguré au milieu des années 2000, qui correspond à une mobilisation des budgets de l'aménagement du territoire en faveur des territoires susceptibles d'être moteurs au niveau national dans une perspective de compétition internationale, comme l'illustre la politique des pôles de compétitivité.

Ainsi, alors même que les villes moyennes constituent un ensemble de villes important au sein du réseau urbain français, penser aujourd'hui leur rôle en matière d'aménagement du territoire appa-

17. Il est fait référence ici à l'appel à expérimentation lancé par la DIACT en 2007. Les villes moyennes concernées par l'expérimentation dans le domaine de l'enseignement supérieur et de la recherche sont : Albi, Auch, Bourges, Mâcon, Montbéliard, Roanne, Saint-Brieuc, Tarbes, Troyes (voir le site Internet dédié : [http://www.villesmoyennestemoins. $\mathrm{fr} / \mathrm{]})$

18. Selon le compte-rendu de la réunion de synthèse qui s'est tenu le 11 juin 2009. Toutefois, la Région et l'État ont été amenés à se positionner dans le cadre des schémas locaux d'enseignement supérieur et de recherche en région Rhône-Alpes d'une part, et dans celui du PRES en région Midi-Pyrénées, d'autre part. raît comme une opération malaisée. L'intérêt nouveau qu'elles suscitent (DIACT, 2007), même s'il apparaît en retrait par rapport aux grands objectifs contemporains de l'aménagement du territoire, amène à s'interroger sur la situation actuelle de ces villes au regard des enjeux d'aménagement du territoire afin d'en proposer une conception renouvelée pour l'action.

\section{VERS UNE CONCEPTION RENOUVELÉE DES VILLES MOYENNES POUR L'AMÉNAGEMENT DU TERRITOIRE}

\section{Les enjeux des villes moyennes au regard de l'aménagement du territoire}

Au sein du territoire français, les villes moyennes constituent un ensemble de poids tant d'un point de vue démographique que de celui de leur rôle dans la structuration de l'espace. Considérées à travers les aires urbaines de 30000 à 200000 habitants, les villes moyennes représentent plus de $25 \%$ de la population de l'ensemble des aires urbaines (plus de 45 millions d'habitants) au recensement de 1999. Elles correspondent à 156 aires (soit le quart des aires urbaines françaises) regroupant plus de 11 millions d'habitants (DIACT, 2007).

Les villes moyennes constituent un semis dense de villes réparties de manière assez homogène sur le territoire national (figure 2) et participent de manière variée à sa structuration. Tout d'abord, certaines villes moyennes, incluses dans des espaces dominés par de grandes agglomérations, participent à l'organisation et à la vie de relations des espaces métropolitains. Au contraire, du fait de leur isolement relatif vis-à-vis des centres de taille plus importante, d'autres villes moyennes vivent dans une certaine autonomie (Limoges, Angoulême, Niort, Nevers, Aurillac, Cherbourg et Bastia). D'autres organisent, grâce aux relations qu'elles entretiennent, des espaces à l'échelle d'un département, voire d'une petite région (Besançon, Caen, Pau, La Rochelle, Agen). Enfin, des villes moyennes placées dans la dépendance d'une grande ville polarisent à leur tour d'autres villes moyennes (Annecy vis-à-vis d'Annemasse, de Thonon-les-Bains, de Cluses et de Sallanches). Par conséquent, ces villes peuvent se trouver dans la dépendance marquée vis-à-vis d'une grande agglomération ou, à l'inverse, elles peuvent 


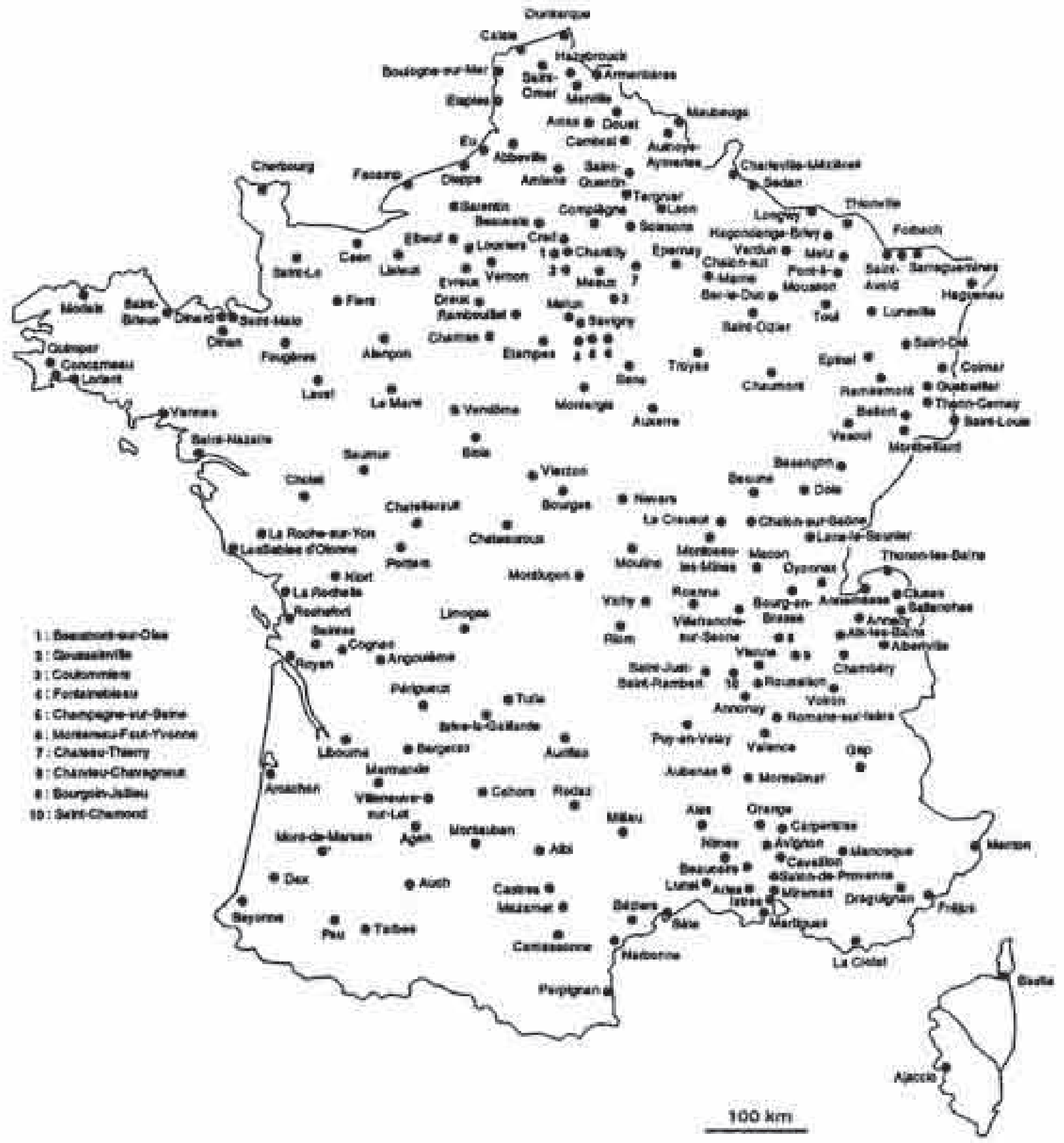

Figure 2 : Le semis des villes moyennes - unités urbaines de 20000 à 200000 habitants en 1990 (source : Santamaria, 1999) The spatial distribution of medium-sized cities in 1990

avoir un rôle d'organisation de l'espace à une échelle quasi-régionale. Dans ces deux cas extrêmes, l'on retrouve ce rôle d'articulation interterritoriale commun à cette catégorie de villes (Santamaria, 1999). Par conséquent, la seule observation du poids et du rôle des villes moyennes au sein du territoire natio- nal rend légitime la question de leur participation active à l'organisation du territoire.

Au-delà de ce simple constat, les enjeux d'aménagement du territoire liés à la situation actuelle des villes moyennes correspondent à des préoccupations qui dépassent cette catégorie de villes. À l'échelle 
locale, les villes moyennes sont directement confrontées à la problématique de la périurbanisation et à celle de l'économie résidentielle. En effet, la dynamique démographique du pourtour périurbain se fait souvent au détriment des communescentres des agglomérations de taille moyenne, voire au détriment de leurs zones centrales, celles-ci continuant à supporter les charges de centralité (DIACT, 2007). De ce point de vue, la situation des villes moyennes est au cœur des débats sur la périurbanisation qui animent les sphères politiques (Grenelle de l'environnement), de l'urbanisme et de l'aménagement (Roux et Vanier, 2009).

Le constat du développement d'une économie résidentielle place aussi cette catégorie de villes au cœur de certains débats relatifs à l'aménagement du territoire. L. Davezies (2008) a montré qu' "Entre 1990 et 1999, les 215 villes moyennes ${ }^{19}$ françaises enregistrent une croissance de leur emploi total privé plus rapide que le reste du pays, ainsi qu'une progression plus forte de leur revenu par habitant ». Cette situation générale s'explique notamment par les apports de l'économie résidentielle $^{20}$. Cependant, l'auteur distingue des cas de figure différents au sein de la catégorie en question : certaines zones d'emploi relevant de villes moyennes participent à la captation de revenus résidentiels qui leur assure une certaine prospérité (Fréjus), d'autres, au contraire, n'y parviennent pas du fait de leur faible attractivité résidentielle souvent liée à une crise de reconversion de l'appareil productif local (Angoulême). Si la situation des villes moyennes apparaît donc contrastée en regard des évolutions constatées ci-dessus, ces dernières amènent à s'interroger sur les ressorts de ce type de développement du territoire national. Ainsi, entre 1993 et 2001, les secteurs les plus créateurs d'emplois, si l'on considère les villes moyennes dans leur ensemble, sont liés à l'économie résidentielle : bâtiment, commerce, administration, services à la personne, éducation, santé, aide sociale dans un contexte de perte d'emploi industriel. Outre les effets de déports de la richesse nationale vers

19. Les critères de choix de ces villes n'étant pas précisés dans le texte de l'auteur, il s'agit vraisemblablement des villes de 20000 à 200000 habitants dans l'unité urbaine en 1999.

20. L'économie résidentielle rassemble les apports des revenus de transferts (base sociale), des salaires de la fonction publique (base publique) et les salaires des actifs travaillant dans une zone d'emploi différente de celle où ils résident, les retraites et les dépenses des touristes. les espaces moins ou peu productifs soulignés par L. Davezies, on peut s'interroger sur le caractère durable de cette situation de rente aussi bien pour les villes moyennes qui bénéficient de cette situation $^{21}$ que pour l'ensemble du territoire national.

Le constat dressé à partir des réflexions sur l'économie résidentielle concerne également la captation par le milieu rural d'une partie de la richesse nationale (Talandier, 2007). Or, cette situation pose le problème des rapports des villes moyennes avec ces nouveaux espaces. En effet, à plus ou moins long terme, on peut imaginer que les villes moyennes auront à supporter de nouvelles charges de centralité liées aux exigences des habitants installés dans les espaces ruraux de proximité au-delà des couronnes périurbaines. On peut également assister à une forme de déconnexion plus radicale entre villes moyennes et espaces ruraux, ces derniers bénéficiant d'une certaine autonomie liée au développement de l'économie résidentielle d'une part, et s'inscrivant dans des relations directes avec les grandes villes grâce à la forte mobilité de leurs habitants, d'autre part.

Toujours à l'échelle locale, et en lien avec les questions relatives à la périurbanisation et aux rapports avec le milieu rural environnant, se pose également la question de la capacité de ces villes à organiser l'espace autour d'elles, notamment en termes d'intercommunalité et de planification spatiale (SCoT). Ici, l'enjeu est celui de l'identification de territoires comme niveau de régulation infranational (Corroleur et Pecqueur, 1996). Il s'agit donc d'un enjeu de gouvernance locale, qui du fait de l'importance des villes moyennes dans la structuration du territoire français et dans l'organisation de l'intercommunalité urbaine, prend une dimension d'enjeu à l'échelle nationale, notamment au titre de la coordination horizontale mais aussi verticale des politiques sectorielles.

À l'échelle régionale, voire macro-régionale, c'est la capacité des villes moyennes à participer à la structuration d'espaces régionaux plus ou moins métropolisés qui est en question. La métropolisation est souvent considérée comme un prolongement du processus classique de concentration que repré-

21. Il faut également considérer les effets de sélectivité entre les villes du fait de leur positionnement plus ou moins favorable du point de vue de l'économie résidentielle. L'économie résidentielle peut alors produire des effets identiques à ceux de la compétition entre villes. 
sente la ville en général. La spécificité de ce phénomène réside dans l'observation d'un accroissement du poids des plus grandes villes dans la répartition de certaines fonctions, ainsi que la concentration de la population dans des aires métropolitaines. Le processus métropolitain s'appuie alors sur une mise en réseau des principales agglomérations dans laquelle les phénomènes de connectivité tendent à l'emporter sur les relations de proximité (Elissalde, page consultée le 30 septembre 2010). Cependant, cette évolution pose la question du rapport des métropoles à leur territoire régional voire macro-régional. Elle laisse apparaître le risque d'une relative déconnexion des territoires environnants et, notamment, des villes moyennes situées dans l'orbite des métropoles. L'enjeu est donc celui de l'organisation de vastes espaces régionaux et macro-régionaux au-delà de la formation de structures discontinues et hétérogènes, faisant alterner en périphérie des zones de faibles densités et des centralités secondaires (edgescities, centres d'affaires, technopoles, etc.) conduisant à la formation d'espaces urbains de plus en plus fragmentés.

Dans le cas français, seule la région Lyonnaise semble pouvoir justifier d'une organisation métropolitaine s'appuyant sur les villes moyennes situées dans l'orbite de Lyon. Bonneville (1993) a montré que « La réorganisation des espaces n'obéit plus à l'opposition centre-périphérie mais se recompose sur un mode réticulaire, redéfinissant les articulations urbaines selon des relations fonctionnelles ou culturelles à différentes échelles ». Les villes moyennes directement concernées par ce phénomène sont celles appartenant à la Région Urbaine de Lyon voire, au-delà, celles situées dans un rayon de 80 kilomètres autour de la métropole régionale (Bonneville, 1997).

Dans le cas de Paris et de l'Île-de-France, au cours de ces dernières années, on a assisté à la diffusion dans l'espace régional de l'emploi. Ceci peut être considéré comme un rééquilibrage entre Paris et les autres parties de l'espace régional au profit de ce dernier. Mais, alors que certains y voient une évolution positive permettant de rapprocher emplois et logements, d'autres, au contraire, à l'instar de Davezies, considèrent que cette évolution limite, du fait de la dispersion des emplois, les capacités d'accès des franciliens à un travail. Des choix politiques favorisant une reconcentration de l'emploi au centre accompagnée d'une politique de transports assurant un meilleur accès des franciliens à ces emplois, quel que soit leur lieu d'habitation ${ }^{22}$, semble donc devoir être privilégiés. Cependant, prenant en compte la situation de fait tout en tentant de la dépasser, on peut également envisager l'aménagement de la région Île-de-France sur le registre d'une organisation de centres en réseau jouant de spécialisation, de complémentarité et de connectivité ${ }^{23}$. Ces évolutions et ces choix concernent les petites villes et l'espace rural de la région mais aussi les villes moyennes régionales ainsi que celles situées au sein de l'aire urbaine de Paris.

À l'échelle nationale et européenne, les villes moyennes sont concernées par la quête d'une organisation plus équilibrée du territoire via le recours à la notion de polycentrisme. Cette notion constitue une forme de reviviscence récente des objectifs initiaux de la politique d'aménagement du territoire en France à travers la recherche d'une organisation plus équilibrée du territoire national mais également européen.

Introduite par l'Union européenne à travers le Schéma de développement de l'espace communautaire (1999), elle constitue une réponse au constat selon lequel le développement du grand marché européen fondé sur la libre circulation des personnes, des biens et des capitaux risque de se traduire par la concentration dans l'espace des richesses et des populations. Ainsi, le polycentrisme est présenté dans le SDEC comme une orientation visant à contrebalancer le caractère mono-centrique du territoire européen, organisé autour du Pentagone Londres-Paris-Milan-Munich-Hambourg. Elle doit aboutir, via l'action de l'Union notamment, à une conception plus équilibrée du développement du

22. Dans un article en ligne sur le site UrbaPlus de l'Institut d'urbanisme de Paris, L. Davezies qualifie la solution de reconcentration de l'emploi de «petit enfer », la situation de dispersion de l'emploi, de " grand enfer» : «La périurbanisation des logements et la concentration des emplois au centre, également accessibles aux actifs de tous les quadrants via des transports radiaux économiques et amicaux avec l'environnement ne pose que des problèmes de consommation d'espace. C'est le "petit enfer". L'étalement simultané des actifs et des emplois ne garantit pas que pour un actif donné, l'emploi qui l'intéresse se localise près de chez lui. Si cet emploi se localise dans d'autres quadrants, il n'y a plus accès ou seulement, pour les plus proches, en voiture. La solution "à l'américaine" consistant à déménager pour se rapprocher de l'emploi est exclue du fait des "artefacts" que constituent les freins à la mobilité résidentielle que l'on soit propriétaire ou locataire social ou privé. C'est le "grand enfer". » L. Davezies, (page consultée le 7 octobre 2010).

23. Une telle situation a pu être constatée dans le cas, comparable, des medium-sized cities du bassin de Londres qui ont participé aux dynamiques économiques et démographiques de cet espace dans les années 80-90 (Santamaria, 1999). 
territoire européen permettant d'atteindre l'objectif de cohésion territoriale ${ }^{24}$. À ce titre, le polycentrisme permet d'envisager la promotion de pôles au sein des espaces périphériques.

Selon l'analyse de Allain (2002), le polycentrisme correspond, à l'échelle européenne, à la mise en place de réseaux de villes dont aucune ne possède la masse critique pour jouer un rôle de centre. Ce qui est visé ici, c'est la coopération transeuropéenne de villes qui peuvent être très éloignées collaborant sur des objectifs limités: Allain cite l'exemple de Rennes et de Hambourg dans le domaine de la recherche électronique. Dans une autre acception, "Le polycentrisme peut signifier réseau urbain réticulé - c'est l'auteur qui souligne - où les points de connexion des mailles intermédiaires sont de niveau comparable sur le plan hiérarchique : les mailles pouvant correspondre à des aires d'une surface équivalente à celle d'un petit État ou d'une grande région (Belgique, Andalousie, Bavière). Ce qui sousentendrait qu'un grand pôle pour chacune de ces régions satisferait aux exigences d'un équilibre du territoire européen » (Allain, 2002). Cependant, ces " mailles intermédiaires », bien que dominées par un pôle s'organiseraient elles-mêmes sur un modèle polycentrique comme dans le cas de la Randstad Holland: Delft, Gouda, Hilversum, par exemple, sont des villes comparables en taille aux villes moyennes françaises. Enfin, le polycentrisme peut également correspondre à une volonté de soutenir le développement local en dynamisant des réseaux à petites mailles intra-régionales censés résister aux tendances polarisatrices de la globalisation.

Dans le cas français, si on voit mal les villes moyennes participer à une mise en réseau à l'échelle européenne (la coopération entre grandes villes n'ayant elle-même rien de facile car dépendante de l'efficacité des réseaux et de la volonté de coopérer), elles pourraient plus facilement trouver leur place dans des organisations régionales et dans les dynamiques de développement local telle que prévues par le polycentrisme européen (ESPON, 2006). En

24. La cohésion territoriale correspond à l'idée d'une solidarité qui s'exprime entre les territoires de l'Union via la mise en œuvre de la politique régionale. De ce point de vue, cette expression peut être référée à un des objectifs historiques de l'Europe : la diminution des disparités, des écarts et des déséquilibres entre les États et entre les régions européennes afin de permettre la réalisation du marché intérieur et l'amélioration de la qualité de vie de tous les citoyens européens. Elle exprime également l'ambition de garantir un cadre de vie et des opportunités identiques quelle que soit la localisation en Europe.
France, une telle perspective pose la question de la planification spatiale multi-échelle. Certes, la tradition d'aménagement du territoire en France, qui reste plutôt centrée sur une approche en termes de développement économique régional (Union européenne, Direction générale de la politique régionale et de la cohésion, 2000), a connu une évolution vers une approche en termes de planification spatiale multi-échelle. Cependant, ce mouvement reste partiel et incertain. En effet, c'est surtout à l'échelle locale, dans le domaine de l'urbanisme, qu'elle s'exprime à travers l'articulation entre les schémas de cohérence territoriale et les plans locaux d'urbanisme. À l'échelle régionale, les schémas régionaux d'aménagement du territoire jouent également ce rôle mais ils apparaissent avant tout comme des documents politiques sans portée normative; leur vocation étant de nourrir la contractualisation avec l'État. À l'échelle nationale, les schémas de services collectifs instaurés par la loi d'orientation pour l'aménagement et le développement durable du territoire du 25 juin 1999 sont aujourd'hui tombés en désuétude. Pour leur part, les directives territoriales d'aménagement ne concernent que certaines parties du territoire national jugées «stratégiques » par l'État. Du point de vue de l'aménagement du territoire, la question du polycentrisme met donc en jeu la capacité à penser une organisation multi-échelle du territoire au sein de laquelle les villes moyennes peuvent avoir leur place, notamment aux échelles régionales et locales. Considéré à l'échelle nationale, cela passe sûrement par une réflexion en termes de planification spatiale qui couvre toutes les échelles de l'aménagement. Il s'agirait de prendre en compte les différents types d'organisations territoriales infranationales auxquelles participent les villes moyennes et leur articulation à l'échelle nationale.

Porteuses d'enjeux transversaux de l'aménagement à différentes échelles, on peut s'étonner du manque d'intérêt que suscitent les villes moyennes. Celui-ci peut s'expliquer par la difficulté à considérer ces villes en dehors d'une catégorie qui met en avant le caractère subalterne de ces villes.

\section{Les villes moyennes : des villes subalternes}

La catégorie «ville moyenne » renvoie à la définition, par la géographie, de hiérarchies urbaines 
permettant, notamment, de distinguer différents niveaux de villes en fonction de leur taille et de leur rôle dans l'espace. Généralement, en France, le réseau urbain n'est pas regardé comme un système composé de sous-systèmes variés, mais comme une organisation au caractère fortement hiérarchique et dotée d'une forte inertie. Cette approche dessine dans l'espace une hiérarchie fonctionnelle fondée sur la théorie des lieux centraux énoncée par Christaller. Elle est à l'origine de nombreux travaux de géographie qui se développent en France à partir des années 1950-1960. Les recherches s'orientent alors vers l'étude de la hiérarchie des villes. Cette démarche fait apparaître une grande stabilité dans le temps de la structure hiérarchique du réseau urbain. Ce dernier est alors conçu comme l'expression conceptuelle de l'inertie des villes dans leurs rapports hiérarchiques. Or, ce qui ne devrait être qu'une forme de représentation de la réalité - l'observation de la structure hiérarchique des villes entre-elles - s’impose comme la réalité elle-même dès lors qu'il s'agit seulement d'étudier la hiérarchie, les « déviances » par rapport au modèle pouvant soit y être incorporées, soit être rejetées comme non pertinentes. C'est notamment l'enjeu de l'utilisation plus répandue, à partir de la fin des années 1980, du terme de «système urbain » qui permet d'incorporer les « déviances » tout en maintenant l'idée d'une forte capacité de reproduction. On constate donc la force du paradigme du réseau urbain fondée sur une approche hiérarchique. Cette dernière débouche sur une mise en catégorie des villes qui identifie, notamment, une catégorie de villes moyennes située entre celle des grandes villes et celle des petites villes... Les villes moyennes sont donc définies par leur appartenance à une catégorie de taille qui les place dans une situation hiérarchique spécifique ${ }^{25}$. Or, l'approche par la hiérarchie urbaine fait peu de cas des villes qui composent cette catégorie au moment de l'observation, de leurs éventuelles spécificités voire, en considérant des périodes de temps plus longues, de leurs trajectoires spécifiques; elle

25. Cette mise en catégorie scientifique, même si elle n'est pas spécifique à la France, n'est pas systématiquement mobilisée dans le champ de la géographie. Au Royaume-Uni, par exemple, l'expression medium-sized cities s'apparente à une facilité de langage qui désigne, selon les cas, des villes dont la population de l'aire urbaine est comprise entre 25000 et 120000 habitants et qui jouent le rôle de centre urbain d'une région non dominée par une des plus grandes agglomérations du pays (Santamaria, 2000). écarte la question des relations entre villes qui ne suivraient pas le schéma hiérarchique.

Cette approche scientifique se double d'une représentation ordinaire congruente de la ville moyenne considérée comme la ville qui permettrait de disposer des avantages de la vie urbaine incarnée par la grande ville sans subir les inconvénients de la vie dans les petites villes. En effet, la dimension modeste de ces villes faciliterait les déplacements et les contacts entre les personnes. Les habitants disposeraient d'un cadre de vie privilégié, celui de la ville elle-même, et de la nature, toujours proche. En outre, les rapports sociaux y seraient plus pacifiques que dans les grandes villes. Dans un article, Michel (1977) constate que la ville moyenne fait l'objet d'« une description affective et flatteuse, d'où elle ressort parée d'attraits, de qualités, de vertus. Les maîtres-mots en sont l'agrément, le charme, la discrétion, la modestie, l'humanité, l'harmonie, l'équilibre... On vante son charme, sa grâce. On la trouve accueillante, agréable à vivre. On s'y sent tranquille, en sécurité » (p. 657). Dans les années 1970, quand l'État entreprend de mettre en œuvre une politique des villes moyennes, il contribue à promouvoir une image concordante de ces villes. La ville moyenne aurait donc les qualités de ce qui la définit comme «moyenne » : la modération, la douceur de vivre, l'équilibre, l'humanité, l'harmonie, la sécurité. Dans un sondage effectué par la SOFRES pour le compte de la Fédération des maires des villes moyennes (FMVM) en 2005 à partir d'un échantillon représentatif de la population des villes moyennes, ces dernières sont perçues par leurs habitants comme des villes agréables à vivre, disposant d'une qualité de vie appréciée ${ }^{26}$. Sont notamment valorisés par les personnes interrogées la qualité du cadre urbain et le sentiment d'être en sécurité. Cependant, les qualités reconnues aux villes moyennes peuvent également faire l'objet d'une analyse en creux. Des caractéristiques positives peuvent alors se transformer en défauts : la modération devient un manque d'imagination, la douceur de vivre de l'endormissement, l'équilibre de l'ennui, l'harmonie du conformisme (Gault, 1989). Le caractère ambigu des "qualités » que l'on prête aux villes moyennes rend cette catégorie

26. Enquête réalisée par la SOFRES pour le compte de la Fédération nationale des maires des villes moyennes (téléchargeable: [www.villesmoyennes.asso.fr/upload/document/doc_201001150315200.pdf]). 
sensible aux choix politiques ${ }^{27}$. Dans un contexte où l'aménagement s'oriente vers la promotion de la compétitivité du territoire national en tentant d'identifier des territoires d'excellence, il est peu étonnant que les villes moyennes, qui représentent une forme sinon affaiblie du moins ambiguë d'urbanité, n'apparaissent pas comme une catégorie prioritaire de l'action publique...

Ainsi, la combinaison entre l'approche scientifique visant à identifier une catégorie moyenne de la hiérarchie urbaine, la représentation ordinaire de la ville moyenne et les nécessités d'une mise en catégorie au nom d'une action publique dédiée, produisent l'image faussée d'un ensemble homogène de villes. Or, cette catégorie correspond, comme nous l'avons signalé auparavant, à des situations variées tant d'un point de vue économique que géographique. Cependant, du point de vue de l'aménagement du territoire, reste le problème de la prise en compte d'une catégorie de villes dont on conteste l'existence sur la base de la variété des réalités qu'elle recouvre! C'est donc la catégorie «ville moyenne » qui doit maintenant être interrogée.

\section{Transcender la catégorie pour l'action : d'une catégorie descriptive à une catégorie renouvelée de l'action publique}

Je fais ici mienne l'idée de R. Brunet selon laquelle les villes moyennes sont " un objet réel non identifié » (Brunet, 1997, p. 188). « Réel », parce qu’il existe une incontestable réalité : celle de l'existence d'une catégorie de villes qui ne s'apparente ni à des centres d'intérêt strictement local, ni à des agglomérations disposant d'un véritable rôle d'organisation régionale. "Non identifié », car les limites de taille et les fonctions de la catégorie varient selon les chercheurs, les organismes qui étudient ces villes et les contextes géographiques d'étude ${ }^{28}$. Dans ce sens, la catégorie des villes moyennes devient plus volontiers une facilité de langage permettant de faire référence à des réalités variées. Cette proposition

27. Nous entendons par « choix politiques », les choix effectués par les gouvernements en vertu d'une argumentation prenant en compte des éléments de contexte et proposant des solutions qui semblent de nature à accompagner ou à contrebalancer des évolutions présentées comme inéluctables. Ces choix sont fondés sur un ou plusieurs postulats qui permettent d'orienter la décision dans le sens souhaité (Denquin [1985]).

28. Santamaria, 1999, « La ville moyenne comme centre micro-régional une réalité ambiguë ». n’invalide donc pas l'idée d'avoir recours à la catégorie « ville moyenne » mais permet d'apprécier la diversité des villes qui composent la catégorie tant d'un point de vue statique (les différentes situations au temps t) que dynamique (les évolutions de chaque ville). Elle permet également de replacer ces villes dans la perspective d'enjeux ( $c f$. ceux présentés au point 3.1.) qui ne concernent pas seulement la catégorie et qui varient en fonction de la diversité des situations de ces villes. Ce regard sur l'action d'aménagement du territoire en direction des villes moyennes devrait permettre de combiner, selon les propositions formulées en 2005 par la coopérative ACADIE dans le cadre d'une étude pour la DATAR (ACADIE, 2005), un traitement catégoriel, singulier et systémique de ces villes. Catégoriel, car les villes moyennes partagent des problématiques communes liées à leur taille et aux déstabilisations induites par les évolutions du territoire national sous l'effet de la mondialisation. L'action publique peut ici passer par une aide visant à renforcer les fonctions de centralité au niveau intermédiaire qui est celui des villes moyennes ou encore par un appui à la capacité d'ingénierie publique, notamment dans une perspective de pilotage de la gouvernance territoriale locale. Systémique, tant il est vrai que les villes moyennes sont dépendantes des espaces régionaux au sein desquels elles s'insèrent. Dans une perspective de définition de politiques publiques, cela devrait conduire à développer des études de géographie régionale permettant d'évaluer le potentiel et les conditions d'un développement local/régional auquel les villes moyennes pourraient participer. Singulier, enfin, car les trajectoires, notamment économiques des villes moyennes, ne sont pas identiques, ce qui justifient des interventions au cas par cas. Si ces propositions constituent un modus operandi, les actions concrètes restent encore aujourd'hui à établir.

\section{Conclusion}

La catégorie « ville moyenne » apparaît pour partie obsolète du point de vue de la conception d'une action d'aménagement du territoire. Non seulement parce que l'on s'accorde sur la variété des situations et des évolutions de ces « objets réels non identifiés » sur le territoire national mais aussi parce que l'entrée catégorielle réduit les enjeux de ces villes à ceux de la catégorie elle-même. Or, comme nous 
l'avons montré, les enjeux des villes moyennes sont également des enjeux d'aménagement du territoire qui concernent les territoires à différentes échelles du local à l'Europe. Bâtir des actions concrètes au nom de l'aménagement du territoire pour ces villes reste un chantier qui ne pourra déboucher, selon nous, qu'en fonction de choix politiques répondant aux enjeux identifiés. Les travaux menés actuellement par la DATAR dans le cadre de l'expérimentation en villes moyennes d'une part ${ }^{29}$, et ceux de prospective sur les villes intermédiaires ${ }^{30}$ pourront peut-être y aider.

\section{Bibliographie}

Datar, Acadie, 2005. Les villes moyennes : enjeux d'action publique, DATAR/ACADIE, Paris, 23 p.

Allain R., 2002. Du concept au projet polycentrique, dans Baudelle G., Castagnède B. (dir.), Le polycentrisme en Europe, DATAR, Édition de l'aube, La Tour d'Aigues, p. 181-211

Bonneville M., 1997. Lyon, Métropole régionale ou euro-cité?, Anthropos, Paris, 202 p.

Bonneville M., 1993. Processus de métropolisation et dynamiques de recomposition territoriale dans l'agglomération lyonnaise, Métropole en déséquilibre?, Plan urbain / DATAR, Lyon/Paris, Plan/Agence d'urbanisme de la Communauté urbaine de Lyon, Agence d'urbanisme de la Communauté urbaine de Lyon/Économica, p. 321-344.

Braudel F., Labrousse E., 1993. Histoire économique et sociale de la France. Tome IV, Paris, PUF, 1847 p.

Brunet R., 1997. Territoires de France et d'Europe (Raisons de géographe), Paris, Belin, 319 p.

Brunet R., 1989. Les villes « européennes », Paris, La Documentation française, $79 \mathrm{p}$.

Commerçon N., 1990. Les habits neufs de la ville moyenne, Colloque ASDRLF, Multigraphié, Saint-Étienne, 3-4-5 septembre, 9 p.

Corroleur F., Pecqueur B., 1996. Les politiques économiques locales en France durant la décennie 80. La construction de spécificités territoriales comme enjeu stratégique pour les collectivités locales, dans Demazières C. (dir.), Du local au global, les initiatives locales pour le développement économique en Europe et en Amérique, Paris, L'Harmattan, $382 \mathrm{p}$.

DATAR, 1995. La politique des réseaux de villes. Colloque Quel avenir pour les réseaux de villes?, multigraphié, s. éd., s. l.

DAvezies L., 2008. La république et ses territoires. La circulation invisible des richesses, La République des idées, Seuil, Paris, $110 \mathrm{p}$.

29. Cf. supra note 23.

30. Il est fait ici référence au programme de prospective de la DATAR, « Prospective 2040 » et notamment aux travaux sur « les villes intermédiaires et leurs espaces de proximité ».
DAvezies L., (page consultée le 7 octobre 2010). Croissance sans développement en Île-de-France [en ligne], adresse URL : [www.urbaplus.org/.../Rapport_Davezies_CDC_IDF_croissancesansdeveloppement.pdf].

Denquin J.-M., 1985. Science politique, PUF, Paris, 453 p.

DIACT, 2007. Les villes moyennes françaises. Enjeux et perspectives, DIACT, Paris, 59 p.

Dodier R., 1995. L'évolution récente de systèmes fordistes: l'exemple des villes moyennes des pays de la Loire, Colloque Les villes moyennes dans l'Arc atlantique, multigraphié, Cholet, 18-19 avril, 10 p.

Dumas J., 1991. Planification économique et aménagement urbain : politiques de la ville ou politiques des villes françaises, L'État et les stratégies du territoire, dans THÉRY H. (dir.), CNRS, PARIS, p. 157-165.

Elissalde B., (page consultée le 30 septembre 2010). Métropolisation [en ligne], adresse URL : [http://www.hypergeo.eu/ article.php3?id_article $=75 \#]$

ESPON, 2006. ESPON Project 1.4.1., The role of Small and medium-sized towns (SMETSO), Final Report, Espon Coordination Unit et Österreichisches Institut für Raumplanung (ÖIR), Esch-sur-Alzette, 435 p.

Gault M., 1989. Villes intermédiaires pour l'Europe?, SyrosAlternatives, Paris, 191 p.

Guichard O., 1986. Propositions pour l'aménagement du territoire : rapport au Ministère de l'Équipement, du Logement, de l'Aménagement du Territoire et des Transports, la Documentation française, Paris, $61 \mathrm{p}$.

Lajugie J., 1974. Les villes moyennes, Cujas, Paris, 216 p.

LOINGER G., 1994. Europe 2000 et l'aménagement du territoire, GEISTEL, Paris, 32 p.

Mabileau A., Sorbets C., 1989. Introduction, Gouverner les villes moyennes, dans Mabileau A., Sorbets C. (dir.), CNRS/Pedone, Paris, p. 7-19.

Michel M., 1977. Ville moyenne, ville-moyen, Annales de géographie, Novembre-Décembre, n 478, Armand Colin/ CNRS, p. 641-685.

Michel M., 1987. La ville moyenne et sa situation géographique, Inter Régions, Novembre, n 96, CNERP, p. 32-44

Pitié J., 1971. Exode rural et migrations intérieures en France: l'exemple de la Vienne et du Poitou-Charentes, Norois, Poitiers, $751 \mathrm{p}$.

Pumain D., Saint-Julien T., 1978. Les dimensions du changement urbain : évolution des structures socio-économiques $d u$ système urbain français de 1945 à 1975, CNRS, Paris, 202 p.

Roux E., Vanier M., 2008. La périurbanisation, La documentation française, Paris, $87 \mathrm{p}$.

SAllez A., 1992. Les nouveaux territoires de l'entreprise, dans Derycke P.-H., Espace et dynamiques territoriales, Économica / DATAR, Paris, p. 217-242.

Santamaria F., 1999. Les villes moyennes françaises : entre hiérarchie et réseaux (étude comparée avec l'Espagne et le Royaume-Uni), Presses universitaires du Septentrion, Lille, $513 \mathrm{p}$.

Santamaria F., 2000. La notion de « ville moyenne» en France, en Espagne et au Royaume-Uni, Annales de géographie, $n^{\circ}$ 613, mai-juin, Armand Colin, Paris, p. 227-239. 
TALAndier M., 2007. Un autre modèle de développement hors métropolisation. Le cas du monde rural. Thèse de doctorat soutenue à l'Institut d'Urbanisme de Paris, Paris 12/Val de Marne, sous la direction de L. Davezies. 380 p. + annexes. Tesson F., 1996. Les réseaux de villes en France (recherche sur le rapport de l'élu local à l'espace), Université de Pau et des Pays de l'Adour, 398 p.
Union européenne, Direction générale de la politique RÉGIONALE ET DE LA COHÉSION, 2000. The EU compendium of spatial planning systems and policies. France, Office des publications officielles des communautés européennes, Luxembourg, 151 p. 